\title{
On the existence and essential components of solution sets for systems of generalized quasi-variational relation problems
}

\author{
Nguyen Van Hung and Phan Thanh Kieu*
}

\section{*Correspondence:} ptkieu@dthu.edu.vn

Department of Mathematics, Dong

Thap University, 783 Pham Huu Lau

Street, Ward 6, Cao Lanh City,

Vietnam

\begin{abstract}
In this paper, we study the existence of a solution for a system of quasi-variational relation problems (in short, (SQVR)). Moreover, we discuss the existence of essentially connected components of the solution set for (SQVR). Then the obtained results are applied to systems of quasi-variational inclusions and to systems of weak vector quasi-equilibrium problems. The results presented in the paper improve and extend many results from the literature. Some examples are given to illustrate our results.

MSC: 47J20; 49J40

Keywords: system quasi-variational relation problems; existence; essential components; system quasi-variational inclusion problems; system vector quasi-equilibrium problems
\end{abstract}

\section{Introduction and preliminaries}

Variational relation problems were first introduced and studied by Luc in [1]. These problems include as special cases variational inclusion problems, vector equilibrium problems, vector variational inequality problems and vector optimization problems, etc. Later, the results of many authors had been extended and studied as regards the existence and stability of solutions in different models; see for example [2-11] and the references therein.

In 1950, Fort [12] first introduced the notion of essential fixed points of a continuous mapping from a compact metric space into itself and proved that any mapping can be approximately closed by a mapping whose fixed points are all essential. Later, Kinoshita [13] introduced the notion of essential components of the set of fixed points of a singlevalued map and proved that there exists at least one essential component of the set of its fixed points. Recently, the essential components of the solution set have been studied for vector equilibrium problems [14, 15], vector variational inequality problems [16], etc. Very recently, Yang and $\mathrm{Pu}[17]$ introduced and studied the system of strong vector quasiequilibrium problems (in short, (SSVQEP)) and also obtained the existence of essential components for these problems.

Motivated by the research works mentioned above, in this paper, we introduce the system of generalized quasi-variational relation problems. Then we establish some existence theorems of solution sets for this problem. Moreover, we also obtain an existence theorem for essentially connected components of the set of solutions for a system of generalized 
quasi-variational relation problems. These results are then applied to systems of quasivariational inclusion problems and systems of weak vector quasi-equilibrium problems.

Now, we pass to our problem setting. Let $I=\{1, \ldots, n\}$ be an index set. For each $i \in I$, let $X_{i}$ and $Y_{i}$ be two real locally convex Hausdorff topological vector spaces and $K_{i}$ a nonempty convex compact subset of $X_{i}$. Denote

$$
K_{\hat{i}}=\prod_{j \in I, j \neq i} K_{j}, \quad K=\prod_{i \in I} K_{i}=K_{i} \times K_{\hat{i}}, \quad X=\prod_{i \in I} X_{i} .
$$

For each $x \in K$, we can write $x=\left(x_{i}, x_{\hat{i}}\right)$. For each $i \in I$, let $S_{i}, T_{i}: K \rightarrow 2^{K_{i}}$ be set-valued mappings, and let $R_{i}\left(x_{i}, x_{\hat{i}}, y_{i}\right)$ be a relation linking $x_{i} \in K_{i}, x_{\hat{i}} \in K_{\hat{i}}$ and $y_{i} \in K_{i}$. We consider the following system of generalized quasi-variational relation problems (in short, (SQVR)).

(SQVR): Find $\left(\bar{x}_{i}, \bar{x}_{\bar{i}}\right) \in K$ such that, for each $i \in I, \bar{x}_{i} \in S_{i}(\bar{x})$ and

$$
R_{i}\left(\bar{x}_{i}, \bar{x}_{\hat{i}}, y_{i}\right) \text { holds, } \quad \forall y_{i} \in T_{i}(\bar{x}),
$$

where $\bar{x}$ is a solution of (SQVR). We denote by $\Sigma(R)$ the solution set of (SQVR).

Next, we recall some basic definitions and some of their properties.

Let $X, Y$ be two topological vector spaces; let $A$ be a nonempty subset of $X$ and $F: A \rightarrow$ $2^{Y}$ be a multifunction.

(i) $F$ is said to be lower semicontinuous (lsc) at $x_{0} \in A$ if $F\left(x_{0}\right) \cap U \neq \emptyset$ for some open set $U \subseteq Y$ implies the existence of a neighborhood $N$ of $x_{0}$ such that $F(x) \cap U \neq \emptyset$, $\forall x \in N$.

(ii) $F$ is said to be upper semicontinuous (usc) at $x_{0} \in A$ if, for each open set $U \supseteq G\left(x_{0}\right)$, there is a neighborhood $N$ of $x_{0}$ such that $U \supseteq F(x), \forall x \in N$.

(iii) $F$ is said to be continuous at $x_{0} \in A$ if it is both lsc and usc at $x_{0} \in A$.

(iv) $F$ is said to be closed if $\operatorname{Graph}(F)=\{(x, y): x \in A, y \in F(x)\}$ is a closed subset in $A \times Y$.

Let $A, B, C$ be convex sets in topological vector spaces and $R(x, y, z)$ be a relation between elements of the three sets. The relation $R$ is said to be closed if the set $\{(x, y, z) \in A \times B \times C$ : $R(x, y, z)$ holds $\}$ is closed. The relation $R$ is said to be convex in the first variable if whenever $R\left(x_{1}, y, z\right)$ holds and $R\left(x_{2}, y, z\right)$ holds, then $R\left(\lambda x_{1}+(1-\lambda) x_{2}, y, z\right)$ holds, for all $x_{1}, x_{2} \in A$, $\lambda \in[0,1]$.

Definition 1.1 ([18]) Let $X, Y$ be two topological vector spaces, $A$ is a nonempty subset of $X$, and $F: A \rightarrow 2^{Y}$ be a multifunction; and $C \subset Y$ is a nonempty, closed, and convex cone. $F$ is called upper $C$-continuous at $x_{0} \in A$, if, for any neighborhood $U$ of the origin in $Y$, there is a neighborhood $V$ of $x_{0}$ such that

$$
F(x) \subset F\left(x_{0}\right)+U+C, \quad \forall x \in V .
$$

Definition 1.2 ([18]) Let $X$ and $Y$ be two topological vector spaces and $A$ be a nonempty convex subset of $X$. A set-valued mapping $F: A \rightarrow 2^{Y}$ is said to be properly $C$-quasiconvex if, for any $x, y \in A$ and $\lambda \in[0,1]$, we have

$$
\begin{aligned}
& \text { either } \quad F(x) \subset F(t x+(1-t) y)+C \\
& \text { or } \quad F(y) \subset F(t x+(1-t) y)+C .
\end{aligned}
$$


Lemma 1.1 ([18]) Let $X, Y$ be two Hausdorff topological vector spaces and $F: X \rightarrow 2^{Y}$ be a multivalued map.

(i) If $F$ is upper semicontinuous with closed values, then $F$ is closed.

(ii) If $F$ is closed and $Y$ is compact, then $F$ is upper semicontinuous.

Lemma 1.2 ([19]) Let $X, Y$ be two Hausdorff topological vector spaces and $F: X \rightarrow 2^{Y}$ be a set-valued mapping with compact values. Then $F$ is upper semicontinuous $x_{0} \in X$ if and only if, for each net $\left\{x_{\alpha}\right\} \subseteq X$ which converges to $x_{0} \in X$ and for each net $\left\{y_{\alpha}\right\} \subseteq F\left(x_{\alpha}\right)$, there exist $y_{0} \in F\left(x_{0}\right)$ and a subnet $\left\{y_{\beta}\right\}$ of $\left\{y_{\alpha}\right\}$ such that $y_{\beta} \rightarrow y_{0}$.

Lemma 1.3 ([20]) Let A be a nonempty compact convex subset of a Hausdorff topological vector space $X$. Suppose that $M: A \rightarrow 2^{A} \cup\{\emptyset\}$ be a set-valued map with the following conditions:

(i) for each at $x \in A, M(x)$ is convex;

(ii) for each at $x \in A, x \notin M(x)$;

(iii) for each at $y \in A, M^{-1}(y)=\{x \in A: y \in M(x)\}$ is open in $A$.

Then there exists $x_{0} \in A$ such that $M\left(x_{0}\right)=\emptyset$.

Lemma 1.4 (Kakutani-Fan-Glicksberg [21]) Let A be a nonempty compact convex subset of a locally convex Hausdorff vector topological space X. If $F: A \rightarrow 2^{A}$ is upper semicontinuous and for any $x \in A, F(x)$ is nonempty, convex, and closed, then there exists an $x^{*} \in A$ such that $x^{*} \in F\left(x^{*}\right)$.

\section{Existence of solutions}

In this section, we establish an existence theorem of solutions for system of generalized quasi-variational relation problems.

Theorem 2.1 For each $i \in I$, assume that

(i) $S_{i}$ is upper semicontinuous in $K$ with nonempty compact convex values;

(ii) $T_{i}$ is lower semicontinuous in $K$ with nonempty convex values;

(iii) for all $\left(x_{i}, x_{\hat{i}}\right) \in K_{i} \times K_{\hat{i}}, R_{i}\left(x_{i}, x_{\hat{i}}, x_{i}\right)$ holds;

(iv) the set $\left\{\left(x_{\hat{i}}, y_{i}\right) \in K_{\hat{i}} \times K_{i}: R_{i}\left(\cdot, x_{i}, y_{i}\right)\right.$ does not hold $\}$ is convex in $K_{i}$;

(v) the relation $R_{i}$ is convex in the first variable and closed.

Then the (SQVR) has a solution, i.e., there exists $\left(\bar{x}_{i}, \bar{x}_{\hat{i}}\right) \in K$ such that, for each $i \in I, \bar{x}_{i} \in$ $S_{i}(\bar{x})$ and

$$
R_{i}\left(\bar{x}_{i}, \bar{x}_{\hat{i}}, y_{i}\right) \text { holds, } \quad \forall y_{i} \in T_{i}(\bar{x}) .
$$

Moreover, the solution set of the (SQVR) is closed.

Proof We define a set-valued map: $\Phi: K \rightarrow 2^{K}$ by $\Phi(x)=\prod_{i \in I} \Phi_{i}(x)$, where

$$
\Phi_{i}(x)=\left\{a_{i} \in S_{i}(x): R_{i}\left(a_{i}, x_{i}, y_{i}\right) \text { holds, } \forall y_{i} \in T_{i}(x)\right\}, \quad \forall x \in K .
$$

(The map $\Phi$ is called the best-reply map; see $[14,17]$.)

For each $i \in I$ :

(I) For any $x \in K$, we show that $\Phi_{i}(x) \neq \emptyset$ is nonempty. 
Indeed, for all $x \in K$, we define a set-valued map $M_{i}: S_{i}(x) \rightarrow 2^{S_{i}(x)} \cup\{\emptyset\}$ by

$$
M_{i}(x)=\left\{y_{i} \in T_{i}(x): R_{i}\left(x_{i}, x_{i}, y_{i}\right) \text { does not hold }\right\}, \quad \text { for each } x_{i} \in S_{i}(x) .
$$

(a) For each $x_{i} \in S_{i}(x)$, by condition (iv), $M_{i}(x)$ is a convex set.

(b) For each $x_{i} \in S_{i}(x)$, by condition (iii), $x_{i} \notin M_{i}\left(x_{i}\right)$.

(c) For each $y_{i} \in T_{i}(x)$, by condition (v), the set $M_{i}^{-1}\left(y_{i}\right)=\left\{x_{i} \in S_{i}(x): y_{i} \in M_{i}(x)\right\}=\left\{x_{i} \in S_{i}(x): R_{i}\left(x_{i}, x_{\hat{i}}, y_{i}\right)\right.$ does not hold $\}$ is open in $S_{i}(x)$.

By Lemma 1.3, there exists $\bar{x}_{i} \in S_{i}(x)$ such that $M_{i}\left(\bar{x}_{i}\right)=\emptyset$, i.e., $\Phi_{i}(x) \neq \emptyset$.

(II) We show that $\Phi_{i}(x)$ is convex.

Let $a_{i}^{1}, a_{i}^{2} \in \Phi_{i}(x)$ and $\lambda \in[0,1]$ and put $a_{i}=\lambda a_{i}^{1}+(1-\lambda) a_{i}^{2}$. Since $a_{i}^{1}, a_{i}^{2} \in S_{i}(x)$ and $S_{i}(x)$ is convex, we have $a_{i} \in S_{i}(x)$. Thus, for $a_{i}^{1}, a_{i}^{2} \in \Phi_{i}(x)$, it follows that

$$
R_{i}\left(a_{i}, x_{i}, y_{i}\right) \text { holds, } \quad \forall y_{i} \in T_{i}(x) .
$$

By $(\mathrm{v}), R_{i}$ is convex in the first variable, we have

$$
R_{i}\left(\lambda a_{i}^{1}+(1-\lambda) a_{i}^{2}, x_{i}, y_{i}\right) \text { holds, } \quad \forall \lambda \in[0,1], \forall y_{i} \in T_{i}(x),
$$

i.e., $a_{i} \in \Phi_{i}(x)$. Therefore, $\Phi_{i}(x)$ is convex.

(III) We will prove that $\Phi_{i}$ is upper semicontinuous in $K$ with nonempty compact values. Since $K$ is a compact set, it suffices to show that $\Phi_{i}$ is a closed mapping. Indeed, let $\left\{\left(a_{i}^{\alpha}, x^{\alpha}\right)\right\}_{\alpha \in \Lambda}$ be any a net in $\operatorname{Graph}\left(\Phi_{i}\right)$ such that $\left(a_{i}^{\alpha}, x^{\alpha}\right) \rightarrow\left(a_{i}^{0}, x^{0}\right)$. Now, we need only prove that $a_{i}^{0} \in \Phi_{i}\left(x^{0}\right)$. Since $a_{i}^{\alpha} \in S_{i}\left(x^{\alpha}\right)$ and $S_{i}$ is upper semicontinuous at $x^{0} \in K$ with nonempty compact values, we have $S_{i}$ is closed at $x^{0} \in K$, thus, $a_{i}^{0} \in S_{i}\left(x^{0}\right)$. Suppose to the contrary $a_{i}^{0} \notin \Phi_{i}\left(x^{0}\right)$. Then $\exists y_{i}^{0} \in T_{i}\left(x^{0}\right)$ such that

$$
R_{i}\left(a_{i}^{0}, x_{\hat{i}}, y_{i}^{0}\right) \text { does not hold. }
$$

By the lower semicontinuity of $T_{i}$, there is a net $\left\{y_{i}^{\alpha}\right\}$ with $y_{i}^{\alpha} \in T_{i}\left(x^{\alpha}\right)$ such that $y_{i}^{\alpha} \rightarrow y_{i}^{0}$. Since $a_{i}^{\alpha} \in \Phi_{i}\left(x^{\alpha}\right)$,

$$
R_{i}\left(a_{i}^{\alpha}, x_{\hat{i}}^{\alpha}, y_{i}^{\alpha}\right) \text { holds. }
$$

By condition (v) and (2.2),

$$
R\left(a_{i}^{0}, x_{\hat{i}}, y_{i}^{0}\right) \text { holds. }
$$

This is a contradiction between (2.1) and (2.3). Thus, $a_{i}^{0} \in \Phi_{i}\left(x^{0}\right)$. Hence, $\Phi_{i}$ is upper semicontinuous in $K$ with nonempty compact values.

By the definition of the mapping $\Phi$ is upper semicontinuous with nonempty compact values. By Lemma 1.4, there exists $\left(\bar{x}_{i}, \bar{x}_{\hat{i}}\right) \in K$ such that, for each $i \in I, \bar{x}_{i} \in S_{i}(\bar{x})$ and

$$
R_{i}\left(\bar{x}_{i}, \bar{x}_{\hat{i}}, y_{i}\right) \text { holds, } \quad \forall y_{i} \in T_{i}(\bar{x}) .
$$

(IV) Now we prove that $\Sigma(R)$ is closed. 
Let a net $\left\{x_{\alpha}, \alpha \in I\right\} \in \Sigma(R): x_{\alpha} \rightarrow x_{0}$. We need to prove that $x_{0} \in \Sigma(R)$. Indeed, by the lower semicontinuity of $T_{i}$, for any $y_{i}^{0} \in T_{i}\left(x^{0}\right)$, there exists $y_{i}^{\alpha} \in T_{i}\left(x^{\alpha}\right)$ such that $y_{i}^{\alpha} \rightarrow y_{i}^{0}$. As $x^{\alpha} \in \Sigma(R)$,

$$
R_{i}\left(x_{i}^{\alpha}, x_{i}^{\alpha}, y_{i}^{\alpha}\right) \text { holds. }
$$

Since $S_{i}$ is upper semicontinuous with nonempty and closed values, by Lemma 1.1(i), we find that $S_{i}$ is closed. Thus, $x^{0} \in S_{i}\left(x^{0}\right)$. By condition (v),

$$
R_{i}\left(x_{i}^{0}, x_{\hat{i}}^{0}, y_{i}^{0}\right) \text { holds. }
$$

This means that $x^{0} \in \Sigma(R)$. Thus $\Sigma(R)$ is a closed set.

If we let $S_{i}(x)=T_{i}(x)=K_{i}$ for each $i \in I$ and $x \in X$, then (SQVR) becomes the following system of variational relation problems (in short, (SVR)). So, we obtain following result.

Corollary 2.1 For each $i \in I$, assume that

(i) for all $\left(x_{i}, x_{\hat{i}}\right) \in K_{i} \times K_{\hat{i}}, R_{i}\left(x_{i}, x_{i}, x_{i}\right)$ holds;

(ii) the set $\left\{\left(x_{\hat{i}}, y_{i}\right) \in K_{\hat{i}} \times K_{i}: R_{i}\left(\cdot, x_{\hat{i}}, y_{i}\right)\right.$ does not hold $\}$ is convex in $K_{i}$;

(iii) the relation $R_{i}$ is convex in the first variable and closed.

Then the (SVR) has a solution, i.e., there exists $\left(\bar{x}_{i}, \bar{x}_{\hat{i}}\right) \in K$ such that, for each $i \in I$,

$$
R_{i}\left(\bar{x}_{i}, \bar{x}_{\hat{i}}, y_{i}\right) \text { holds, } \quad \forall y_{i} \in K_{i} .
$$

Moreover, the solution set of the (SVR) is closed.

If $I$ is a singleton, $S_{i}(x)=S(x), T_{i}(x)=T(x)$, then (SQVR) reduces to the following quasivariational relation problem (in short, $(\mathrm{QVR}))$. So, we also obtain the following result.

\section{Corollary 2.2 Assume that}

(i) $S$ is upper semicontinuous in $K$ with nonempty compact convex values;

(ii) $T$ is lower semicontinuous in $K$ with nonempty convex values;

(iii) for all $x \in K, R(x, x)$ holds;

(iv) the set $\{y \in K: R(\cdot, y)$ does not hold $\}$ is convex in $K$;

(v) the relation $R$ is convex in the first variable and closed.

Then the (QVR) has a solution, i.e., there exists $\bar{x} \in K$ such that $\bar{x} \in S(\bar{x})$ and

$R(\bar{x}, y)$ holds,$\quad \forall y \in T(\bar{x})$.

Moreover, the solution set of the (QVR) is closed.

\section{Remark 2.1}

(i) For each $i \in I$, let $X_{i}, Y_{i}$ and $K_{i}, K_{\hat{i}}, K, T_{i}=S_{i}$ be as in (SQVR). Let $F_{i}: K_{i} \times K_{\hat{i}} \times K_{i} \rightarrow 2^{Y_{i}}$ be a set-valued mapping, $C_{i} \subset Y_{i}$ be a nonempty, closed, and convex cone, and the relation $R_{i}$ be defined as follows:

$$
R_{i}\left(x_{i}, x_{\hat{i}}, y_{i}\right) \text { holds } \quad \text { iff } \quad F_{i}\left(x_{i}, x_{\hat{i}}, y_{i}\right) \subset C_{i} \text {. }
$$


Then (SQVR) becomes the system of strong vector quasi-equilibrium problem (in short, (SSVQEP)) studied in [17].

(ii) Let $S_{i}(x)=T_{i}(x)=K_{i}$ for each $i \in I$ and $x \in X$ and let $F_{i}, C_{i}$ as in Remark 2.1(i). Then (SVR) becomes the system of strong vector equilibrium problems (in short, (SSVEP)) studied in [17].

(iii) If $I$ is a singleton, $S_{i}(x)=S(x), T_{i}(x)=T(x)$, and let $F: K \times K \rightarrow 2^{Y}$ be a set-valued mapping, $C \subset Y$ be a nonempty, closed, and convex cone, and the relation $R$ be defined as follows:

$$
R(x, y) \text { holds iff } F(x, y) \subset C .
$$

Then (QVR) becomes the strong vector quasi-equilibrium problem (in short, (SQVEP)) studied in [17].

(iv) Yang and $\mathrm{Pu}$ [17] obtained some existence results for the system of strong vector quasi-equilibrium problems. However, the assumptions of theorems in [17] are different from the assumptions of Theorem 2.1, Corollary 2.1, and Corollary 2.2.

(v) Corollary 2.2 is a particular case of Theorems 3.1 and 3.3 in [2]. However, the assumptions and proof methods in Theorems 3.1 and 3.3 in [2] are different from the assumptions and proof methods of Corollary 2.2.

In the next example all assumptions of Corollary 2.2 are satisfied, but Theorem 3.3 in [17] is not applicable. The reason is that $F$ is neither upper $C$-continuous nor properly $C$-quasiconvex.

Example 2.1 Let $I=\{1\}, X_{i}=Y_{i}=\mathbb{R}, K_{i}=[0,1], C=\mathbb{R}_{+}$, and let $S_{i}, T_{i}: K_{i} \times K_{i} \rightarrow 2^{K_{i}}$ and $F: K_{i} \times K_{i} \rightarrow 2^{Y_{i}}$ be defined by

$$
\begin{aligned}
& S_{i}(x, y)=T_{i}(x, y)=[0,1], \\
& F_{i}\left(x_{i}, x_{i}, y_{i}\right)=F(x, y)= \begin{cases}{[1,2]} & \text { if } x_{0}=\frac{1}{2}, \\
{\left[\frac{1}{2}, 1\right]} & \text { otherwise. }\end{cases}
\end{aligned}
$$

We let the relation $R_{i}$ be defined by $R_{i}\left(x_{i}, x_{\hat{i}}, y_{i}\right)$ holding iff $F_{i}\left(x_{i}, x_{i}, y_{i}\right)=F(x, y) \subseteq \mathbb{R}_{+}$. We show that all assumptions of Corollary 2.2 are satisfied. However, $F$ is neither upper $C$-continuous nor properly $C$-quasiconvex at $x_{0}=\frac{1}{2}$.

Firstly, we prove that $F$ is not upper $C$-continuous at $x_{0}=\frac{1}{2}$. Indeed, we let a neighborhood $U=\left[-\frac{1}{6}, \frac{1}{6}\right]$ of the origin in $Z$, then for any neighborhood $V=\left[\frac{1}{2}-\varepsilon, \frac{1}{2}+\varepsilon\right]$ of $x_{0}=\frac{1}{2}$, where $\varepsilon>0$, we choose $\frac{1}{2} \neq x^{*} \in V$ and $y=\frac{1}{2}$. Then

$$
\begin{aligned}
F\left(x^{*}, y\right) & =F\left(x^{*}, \frac{1}{2}\right)=\left[\frac{1}{2}, 1\right] \not \subset F\left(x_{0}, y\right)+U+C \\
& =F\left(\frac{1}{2}, \frac{1}{2}\right)+\left[-\frac{1}{6}, \frac{1}{6}\right]+\mathbb{R}_{+} \\
& =[1,2]+\left[-\frac{1}{6}, \frac{1}{6}\right]+\mathbb{R}_{+}=\left[\frac{5}{6}, \frac{13}{6}\right]+\mathbb{R}_{+} .
\end{aligned}
$$


Next, we show that $F$ is not properly $C$-quasiconvex at $x_{0}=\frac{1}{2}$. Indeed, we let $y=\frac{1}{2}, \lambda=\frac{1}{2}$, and $x_{1}=0, x_{2}=1$. Then

$$
\begin{aligned}
F\left(x_{1}, y\right) & =F\left(0, \frac{1}{2}\right)=\left[\frac{1}{2}, 1\right] \not \subset F\left(x_{1} \lambda+(1-\lambda) x_{2}, y\right)+C \\
& =F\left(\frac{1}{2}, \frac{1}{2}\right)+\mathbb{R}_{+}=[1,2]+\mathbb{R}_{+}, \\
F\left(x_{2}, y\right) & =F\left(1, \frac{1}{2}\right)=\left[\frac{1}{2}, 1\right] \not \subset F\left(x_{1} \lambda+(1-\lambda) x_{2}, y\right)+C \\
& =F\left(\frac{1}{2}, \frac{1}{2}\right)+\mathbb{R}_{+}=[1,2]+\mathbb{R}_{+} .
\end{aligned}
$$

Thus, it gives also the case where Corollary 2.2 can be applied, but Theorem 3.3 in [17] does not work.

\section{Essential components}

In this section, we discuss the existence of essential components for (SQVR).

First, we recall some notions; see [17, 22, 23]. Let $A$ be a nonempty and compact subset of a linear normed $X$. Denote by $M$ the set of all upper semicontinuous maps $R: A \rightarrow 2^{A}$ with nonempty convex compact values. For any $R, P \in M$, we define $\xi(R, P)=$ $\sup _{x \in A} H(R(x), P(x))$, where $H$ is the Hausdorff metric defined in $A$. It is easy to verify that $(M, \xi)$ is a metric space. For each $R \in M$, we denote by $\Xi(R)$ the set of all fixed points of $R$. By Kakutani-Fan-Glicksberg's fixed point theorem, $\Xi(R)$ is a nonempty compact set.

For each $R \in M$, the connected component of a point $x \in \Xi(R)$ is the union of all the connected subsets of $\Xi(R)$ containing $x$. Note that the connected components are connected closed subsets of $\Xi(R)$, and, since $A$ is compact, thus, all the connected components are connected compact. It is easy to see that the connected components of two distinct points of $\Xi(R)$ either coincide or are disjoint, so that all connected components constitute a decomposition of $\Xi(R)$ into connected pairwise disjoint compact subsets, i.e.,

$$
\Xi(R)=\bigcup_{\alpha \in \Lambda} \Xi_{\alpha}(R)
$$

where $\Lambda$ is an index set. For each $\alpha \in \Lambda, \Xi_{\alpha}(R)$ is a nonempty connected compact subset of $\Xi(R)$, and for any $\alpha, \beta \in \Lambda(\alpha \neq \beta), \Xi_{\alpha}(R) \cap \Xi_{\beta}(R)=\emptyset$.

Definition 3.1 ([22]) Let $R \in M$ and $E$ be a nonempty and closed subset of $\Xi(R)$. $E$ is said to be an essential set of $\Xi(R)$ if, for each open set $O \supset E$, there exists an open neighborhood $U$ of $R$ in $M$ such that for any $R^{\prime} \in U$ with $\Xi\left(R^{\prime}\right) \cap O \neq \emptyset$. If a connected component $\Xi_{\alpha}(R)$ of $\Xi(R)$ is an essential set with respect to $M$, then $\Xi_{\alpha}(R)$ is said be an essential component of $\Xi(R)$ with respect to $M$.

Lemma 3.1 ([23]) For any $R \in M$, there is at least one essential component of $\Xi(R)$ with respect to $M$.

Next, we discuss the existence of essential components for (SQVR). 
Let $\Omega$ be the collection of all (SQVR) satisfying the conditions of Theorem 2.1. For each $\omega \in \Omega$, denote by $\Psi(\omega)$ the solution set of $\omega$. It is easy to see that $\Psi(\omega)=\Xi(\Phi)$, where $\Phi$ is the best-reply map of $\omega$. By the proof of Theorem 2.1, we know $\Phi \in M$.

Definition 3.2 Let $\omega \in \Omega$ and $\Xi_{\alpha}$ a connected component of $\Psi(\omega)$. $\Xi_{\alpha}$ is said to be essential if it, as a connected component of $\Xi(\Phi)$, is an essential component of $\Xi(\Phi)$ with respect to $M$, where $\Phi$ is the best-reply map of $\omega$.

By Lemma 3.1, we obtain the following result.

Theorem 3.1 For any $\omega \in \Omega$, there is at least one essential component of $\Psi(\omega)$.

\section{Remark 3.1}

(i) In the special case of Remark 2.1, Theorem 3.1 improves and extends Theorems 4.1 and 4.2 in [17].

(ii) In 2011, Khanh and Quan [24] studied the existence of essential components for generalized KKM points. Moreover, Khanh and Quan also applied these results to optimization-related problems. However, the assumptions and proof methods are very different from the assumptions and proof methods in Theorem 3.1.

(iii) If $I$ is a singleton, then (SQVR) becomes the variational relation problem (in short, (VR)) studied by Khanh and Luc in [3]. However, Khanh and Luc discussed some kinds of semicontinuous sets as outer-continuous, inner-continuous, inner-open, and outer-open solution sets for (VR), while our Theorem 3.1 discusses the existence of essential components for (SQVR) by using Kakutani-Fan-Glicksberg's fixed point theorem.

\section{Applications (I): system of quasi-variational inclusion problems}

For each $i \in I$, let $X_{i}, Y_{i}$ and $K_{i}, K_{\hat{i}}, K, T_{i}=S_{i}$ be as in (SQVR). Let $F_{i}: K_{i} \times K_{\hat{i}} \times K_{i} \rightarrow 2^{Y_{i}}$ be a set-valued mapping. We consider the following system of quasi-variational inclusion problems (in short, (SQIP)): Find $\left(\bar{x}_{i}, \bar{x}_{i}\right) \in K$ such that, for each $i \in I, \bar{x}_{i} \in S_{i}(\bar{x})$ and

$$
0 \in F_{i}\left(\bar{x}_{i}, \bar{x}_{\hat{i}}, y_{i}\right), \quad \forall y_{i} \in S_{i}(\bar{x}),
$$

where $\bar{x}$ is a solution of (SQIP).

Definition 4.1 Let $X, Y, Z$ be topological vector spaces. Suppose $F: X \times Y \times X \rightarrow 2^{Z}$ is a multifunction. $F$ is said to be generalized $\{0\}$-quasiconvex (in the first variable) in a convex set $A \subset X$, if, whenever $0 \in F\left(x_{1}, y, z\right)$ and $0 \in F\left(x_{2}, y, z\right)$, then $0 \in F\left(\lambda x_{1}+(1-\lambda) x_{2}, y, z\right)$, $\forall x_{1}, x_{2} \in A, \forall \lambda \in[0,1]$.

Theorem 4.1 For each $i \in I$, assume that

(i) $S_{i}$ is continuous in $K$ with nonempty compact convex values;

(ii) for all $\left(x_{i}, x_{\hat{i}}\right) \in K_{i} \times K_{\hat{i}}, 0 \in F_{i}\left(x_{i}, x_{\hat{i}}, x_{i}\right)$;

(iii) the set $\left\{\left(x_{\hat{i}}, y_{i}\right) \in K_{\hat{i}} \times K_{i}: 0 \notin F_{i}\left(\cdot, x_{\hat{i}}, y_{i}\right)\right\}$ is convex in $K_{i}$;

(iv) for all $\left(x_{\hat{i}}, y_{i}\right) \in K_{\hat{i}} \times K_{i}, F_{i}\left(\cdot, x_{\hat{i}}, y_{i}\right)$ is generalized $\{0\}$-quasiconvex in $K_{i}$;

(v) the set $\left\{\left(x_{i}, x_{\hat{i}}, y_{i}\right) \in K_{i} \times K_{\hat{i}} \times K_{i}: 0 \in F_{i}\left(x_{i}, x_{\hat{i}}, y_{i}\right)\right\}$ is closed. 
Then the (SQIP) has a solution, i.e., there exists $\left(\bar{x}_{i}, \bar{x}_{\hat{i}}\right) \in K$ such that, for each $i \in I, \bar{x}_{i} \in S_{i}(\bar{x})$ and

$$
0 \in F_{i}\left(\bar{x}_{i}, \bar{x}_{\hat{i}}, y_{i}\right), \quad \forall y_{i} \in S_{i}(\bar{x}) .
$$

Moreover, the solution set of the (SQIP) is closed.

Proof Let the relation $R_{i}$ be defined as follows:

$$
R_{i}\left(x_{i}, x_{\hat{i}}, y_{i}\right) \text { holds } \quad \text { iff } \quad 0 \in F_{i}\left(x_{i}, x_{\hat{i}}, y_{i}\right) .
$$

Then the problem (SQIP) becomes a particular case of (SQVR) and Theorem 4.1 is a direct consequence of Theorem 2.1.

Next, we discuss the existence of essential components for (SQIP).

Let $\Theta$ be the collection of all (SQIP) satisfying the conditions of Theorem 4.1. For each $\theta \in \Theta$, denote by $\Delta(\theta)$ the solution set of $\theta$. It is easy to see that $\Delta(\theta)=\Xi(\Phi)$, where $\Phi$ is the best-reply map of $\theta$. By the proof of Theorem 4.1, we know $\Phi \in M$.

Definition 4.2 Let $\theta \in \Theta$ and $\Xi_{\alpha}$ be a connected component of $\Delta(\theta)$. $\Xi_{\alpha}$ is said to be essential if it, as a connected component of $\Xi(\Phi)$, is an essential component of $\Xi(\Phi)$ with respect to $M$, where $\Phi$ is the best-reply map of $\theta$.

By Lemma 3.1, we also obtain the following result.

Theorem 4.2 For any $\theta \in \Theta$, there is at least one essential component of $\Delta(\theta)$.

\section{Applications (II): system of weak vector quasi-equilibrium problems}

For each $i \in I$, let $X_{i}, Y_{i}$ and $K_{i}, K_{\hat{i}}, K, T_{i}=S_{i}$ be as in (SQVR). Let $f_{i}: K_{i} \times K_{\hat{i}} \times K_{i} \rightarrow Y_{i}$ be a vector function and $C_{i} \subset Y_{i}$ be a nonempty, closed, and convex cone. We consider the following weak system of quasi-equilibrium problems (in short, (WSQVEP)).

(WSQVEP): Find $\left(\bar{x}_{i}, \bar{x}_{\hat{i}}\right) \in K$ such that, for each $i \in I, \bar{x}_{i} \in S_{i}(\bar{x})$ and

$$
f_{i}\left(\bar{x}_{i}, \bar{x}_{\hat{i}}, y_{i}\right) \notin-\operatorname{int} C_{i}, \quad \forall y_{i} \in S_{i}(\bar{x}) .
$$

Definition 5.1 Let $X, Y, Z$ be topological vector spaces and $C \subset Z$ be a nonempty, closed, and convex cone. Suppose $f: X \times Y \times X \rightarrow Z$ is a vector function. $f$ is said to be weakly $C$-quasiconvex (in the first variable) in a convex set $A \subset X$, if whenever $f\left(x_{1}, y, z\right) \notin-\operatorname{int} C$ and $f\left(x_{2}, y, z\right) \notin-\operatorname{int} C$, then $f\left(\lambda x_{1}+(1-\lambda) x_{2}, y, z\right) \notin-\operatorname{int} C, \forall x_{1}, x_{2} \in A, \forall \lambda \in[0,1]$.

Theorem 5.1 For each $i \in I$, assume that

(i) $S_{i}$ is continuous in $K$ with nonempty compact convex values;

(ii) for all $\left(x_{i}, x_{\hat{i}}\right) \in K_{i} \times K_{\hat{i}}, f_{i}\left(x_{i}, x_{\hat{i}}, x_{i}\right) \notin-\operatorname{int} C_{i}$;

(iii) the set $\left\{\left(x_{\hat{i}}, y_{i}\right) \in K_{\hat{i}} \times K_{i}: f_{i}\left(\cdot, x_{\hat{i}}, y_{i}\right) \in-\operatorname{int} C_{i}\right\}$ is convex in $K_{i}$;

(iv) for all $\left(x_{\hat{i}}, y_{i}\right) \in K_{\hat{i}} \times K_{i}, f_{i}\left(\cdot, x_{\hat{i}}, y_{i}\right)$ is weakly $C_{i}$-quasiconvex in $K_{i}$;

(v) the set $\left\{\left(x_{i}, x_{\hat{i}}, y_{i}\right) \in K_{i} \times K_{\hat{i}} \times K_{i}: f_{i}\left(x_{i}, x_{\hat{i}}, y_{i}\right) \notin-\operatorname{int} C_{i}\right\}$ is closed. 
Then the (WSQVEP) has a solution, i.e., there exists $\left(\bar{x}_{i}, \bar{x}_{i}\right) \in K$ such that, for each $i \in I$, $\bar{x}_{i} \in S_{i}(\bar{x})$ and

$$
f_{i}\left(\bar{x}_{i}, \bar{x}_{\hat{i}}, y_{i}\right) \notin-\operatorname{int} C_{i}, \quad \forall y_{i} \in S_{i}(\bar{x}) .
$$

Moreover, the solution set of the (WSQVEP) is closed.

Proof Let the relation $R_{i}$ be defined as follows:

$$
R_{i}\left(x_{i}, x_{i}, y_{i}\right) \text { holds } \quad \text { iff } f_{i}\left(x_{i}, x_{i}, y_{i}\right) \notin-\operatorname{int} C_{i} \text {. }
$$

Then the problem (WSQVEP) becomes a particular case of (SQVR) and Theorem 5.1 is a direct consequence of Theorem 2.1.

Definition 5.2 ([14]) Let $X$ and $Z$ be two topological vector spaces and $A \subseteq X$ be nonempty convex set, and $C \subset Z$ be a nonempty, closed, and convex cone. Suppose $f: A \rightarrow Z$ is a vector function. $f$ is called $C$-continuous at $x_{0} \in A$ if, for any open neighborhood $V$ of the zero element $\theta$ in $Z$, there exists an open neighborhood $U$ of $x_{0}$ in $A$ such that

$$
f(x) \in f\left(x_{0}\right)+V+C, \quad \forall x \in U
$$

and $C$-continuous in $A$ if it is $C$-continuous at every point of $A$.

Remark 5.1 Lin et al. [14] obtained some existence results of (WSVQEP). However, the assumptions of Theorems 3.1, 3.3, and 3.4 in [14] are different from the assumptions in Theorem 5.1. Example 5.1 shows that all the assumptions of Theorem 5.1 are satisfied. But Theorem 3.1 in [14] does not work. The reason is that $f_{i}$ is not- $C_{i}$-continuous.

Example 5.1 Let $I=\{1\}, X_{i}=Y_{i}=Z_{i}=\mathbb{R}, K_{i}=[0,1], C_{i}=\mathbb{R}_{+}$, and let $S_{i}: K_{i} \rightarrow 2^{K_{i}}$ and $f: K_{i} \rightarrow \mathbb{R}$ be defined by

$$
\begin{aligned}
& S_{i}(x)=[0,1], \\
& f(x, y)=f(x)= \begin{cases}{\left[0, \frac{1}{2}\right]} & \text { if } x_{0}=\frac{1}{6} \\
{[1,2]} & \text { otherwise }\end{cases}
\end{aligned}
$$

We show that all assumptions of Theorem 5.1 are satisfied. However, $f$ is not- $C$-continuous at $x_{0}=\frac{1}{6}$. Thus, it gives the case where Theorem 5.1 can be applied but Theorems 3.1, 3.3, and 3.4 in [14] do not work.

Remark 5.2 If we let $S_{i}(x)=K_{i}$ for each $i \in I$. Then (WSQVEP) becomes the system of vector equilibrium problem (in short, (SVEP)). If we let $I=\{1\}$, then (WSQVEP) becomes the strong vector equilibrium problem (in short, (VEP)). The problems (SVEP) and (VEP) are studied in [14].

Definition 5.3 ([14]) Let $X$ and $Z$ be two topological vector spaces and $A \subseteq X$ be a nonempty convex set, and $C \subset Z$ be a nonempty, closed, and convex cone. Suppose 
$f: A \rightarrow Z$ is a vector function. $f$ is called $C$-quasiconvex-like if, for any $x_{i}, x_{2} \in A$ and each $\lambda \in[0,1]$, we have

$$
\text { either } f\left(\lambda x_{1}+(1-\lambda) x_{2}\right) \in f\left(x_{1}\right)-C
$$

or $f\left(\lambda x_{1}+(1-\lambda) x_{2}\right) \in f\left(x_{2}\right)-C$.

Example 5.2 shows that in the special cases of Remarks 5.1 and 5.2, all the assumptions of Theorem 5.1 are satisfied. But Theorems 3.1, 3.3, and 3.4 in [14] do not work. The reason is that $f_{i}$ is not- $C_{i}$-quasiconvex-like.

Example 5.2 Let $X_{i}, Y_{i}, Z_{i}, K_{i}, C_{i}$ be as in Example 5.1, and let $S_{i}:[0,1] \rightarrow 2^{K_{i}}$ and $f$ : $[0,1] \rightarrow \mathbb{R}$ be defined by

$$
\begin{aligned}
& S_{i}(x)=[0,1] \\
& f(x)= \begin{cases}{\left[\frac{1}{3}, \frac{1}{2}\right]} & \text { if } x=\frac{1}{6} \\
{[2,5]} & \text { otherwise }\end{cases}
\end{aligned}
$$

We show that all assumptions of Theorem 5.1 are satisfied. However, $f$ is not- $C$-quasiconvex-like at $x_{0}=\frac{1}{6}$. Indeed, let $\lambda=\frac{1}{2}$ and $x_{1}=0, x_{2}=\frac{1}{3}$. Then

$$
\begin{aligned}
f\left(\lambda x_{1}+(1-\lambda) x_{2}\right) & =f\left(\frac{1}{6}\right)=\left[\frac{1}{3}, \frac{1}{2}\right] \not \subset f\left(x_{1}\right)+C=f(0)+C \\
& =[2,5]+[0,+\infty), \\
f\left(\lambda x_{1}+(1-\lambda) x_{2}\right) & =f\left(\frac{1}{6}\right)=\left[\frac{1}{3}, \frac{1}{2}\right] \not \subset f\left(x_{2}\right)+C=f\left(\frac{1}{3}\right)+C \\
& =[2,5]+[0,+\infty) .
\end{aligned}
$$

Thus, Theorem 5.1 can be applied but Theorems 3.1, 3.3, and 3.4 in [14] do not work.

Theorem 5.2 Assume for the problem (WSQVEP) assumptions (i), (ii), (iii), and (iv) are as in Theorem 5.1 and replace $(\mathrm{v})$ by $\left(\mathrm{v}^{\prime}\right)$

$\left(\mathrm{v}^{\prime}\right) f_{i}$ is continuous in $K_{i} \times K_{\hat{i}} \times K_{i}$.

Then the (WSQVEP) has a solution. Moreover, the solution set of the (WSQVEP) is closed.

Proof We omit the proof since the technique is similar to that for Theorem 5.1 with suitable modifications.

Next, we discuss the existence of essential components for (WSQVEP).

Let $\Upsilon$ be the collection of all (WSQVEP) satisfying the conditions of Theorem 5.1. For each $\gamma \in \Upsilon$, denote by $\Pi(\gamma)$ the solution set of $\gamma$. It is easy to see that $\Pi(\gamma)=\Xi(\Phi)$, where $\Phi$ is the best-reply map of $\gamma$. By the proof of Theorem 5.1, we know that $\Phi \in M$.

Definition 5.4 Let $\gamma \in \Upsilon$ and $\Xi_{\alpha}$ a connected component of $\Pi(\gamma)$. $\Xi_{\alpha}$ is said to be essential if it, as a connected component of $\Xi(\Phi)$, is an essential component of $\Xi(\Phi)$ with respect to $M$, where $\Phi$ is the best-reply map of $\gamma$. 
By Lemma 3.1, we also obtain the following result.

Theorem 5.3 For any $\gamma \in \Upsilon$, there is at least one essential component of $\Pi(\gamma)$.

Remark 5.3 In the special case of Remarks 5.1 and 5.2, Theorem 5.3 improves and extends Theorems 4.1 and 4.4 in [14].

\section{Competing interests}

The authors declare that they have no competing interests.

\section{Authors' contributions}

All authors contributed equally and significantly in writing this article. All authors read and approved the final manuscript.

\section{Acknowledgements}

The authors thank the editor and the two anonymous referees for their valuable remarks and suggestions, which helped them to considerably improve the article.

Received: 14 February 2014 Accepted: 13 June 2014 Published: 18 Jul 2014

\section{References}

1. Luc, DT: An abstract problem in variational analysis. J. Optim. Theory Appl. 138, 65-76 (2008)

2. Agarwal, RP, Balaj, M, O'Regan, D: A unifying approach to variational relation problems. J. Optim. Theory Appl. 155, 417-429 (2012)

3. Khanh, PQ, Luc, DT: Stability of solutions in parametric variational relation problems. Set-Valued Anal. 16, 1015-1035 (2008)

4. Balaj, M, Lin, LJ: Existence criteria for the solutions of two types of variational relation problems. J. Optim. Theory Appl. $156,232-246(2013)$

5. Latif, A, Luc, DT: Variational relation problems: existence of solutions and fixed points of contraction mappings. Fixed Point Theory Appl. 2013, 315 (2013)

6. Balaj, M, Luc, DT: On mixed variational relation problems. Comput. Math. Appl. 60, 2712-2722 (2010)

7. Yang, Z: On existence and essential stability of solutions of symmetric variational relation problems. J. Inequal. Appl. 2014, 5 (2014)

8. Hung, NV: Continuity of solutions for parametric generalized quasi-variational relation problems. Fixed Point Theory Appl. 2012, 102 (2012)

9. Hung, NV: Sensitivity analysis for generalized quasi-variational relation problems in locally G-convex spaces. Fixed Point Theory Appl. 2012, 158 (2012)

10. Lin, $L$, Ansari, QH: System of quasi-variational relations with applications. Nonlinear Anal. 72, 1210-1220 (2010)

11. Pu, YJ, Yang, Z: Stability of solutions for variational relation problem with applications. Nonlinear Anal. 75, 1758-1767 (2012)

12. Fort, MK: Essential and nonessential fixed points. Am. J. Math. 72, 315-322 (1950)

13. Kinoshita, S: On essential components of the set of fixed points. Osaka J. Math. 4, 19-22 (1952)

14. Lin, Z, Yang, H, Yu, J: On existence and essential components of the solution set for the system of vector quasi-equilibrium problems. Nonlinear Anal. 63, 2445-2452 (2005)

15. Lin, Z, Yu, J: The existence of solutions for the system of generalized vector quasi-equilibrium problems. Appl. Math. Lett. 18, 415-422 (2005)

16. Hou, $\mathrm{SH}$, Gong, $\mathrm{XH}$, Yang, XM: Existence and stability of solutions for generalized Ky Fan inequality problems with trifunctions. J. Optim. Theory Appl. 146, 387-398 (2010)

17. Yang, Z, Pu, YJ: On the existence and essential components for solution set for system of strong vector quasi-equilibrium problems. J. Glob. Optim. 55, 253-259 (2013)

18. Luc, DT: Theory of Vector Optimization. Lecture Notes in Economics and Mathematical Systems. Springer, Berlin (1989)

19. Ferro, F: Optimization and stability results through cone lower semicontinuity. Set-Valued Anal. 5, 365-375 (1997)

20. Yannelis, N, Prabhakar, ND: Existence of maximal elements and equilibria in linear topological spaces. J. Math. Econ. $12,233-245(1983)$

21. Holmes, RB: Geometric Functional Analysis and Its Application. Springer, New York (1975)

22. Hillas, J: On the definition of the strategic stability of equilibria. Econometrica 58, 1365-1390 (1990)

23. Jiang, JH: Essential component of the set of fixed points of the multivalued mappings and its application to the theory of games. Sci. Sin. 12, 951-964 (1963)

24. Khanh, PQ, Quan, NH: Generic stability and essential components of generalized KKM points and applications. J. Optim. Theory Appl. 148, 488-504 (2011)

10.1186/1029-242X-2014-250

Cite this article as: Hung and Kieu: On the existence and essential components of solution sets for systems of generalized quasi-variational relation problems. Journal of Inequalities and Applications 2014, 2014:250 